\title{
circCDYL/microRNA-150-5p participates in modulating growth and migration of colon cancer cells
}

\author{
Wei Cui ${ }^{1}$, Jinhua $\mathrm{Dai}^{2}$, Jianbo $\mathrm{Ma}^{2}$ and $\mathrm{Hao} \mathrm{Gu}^{3}$ \\ ${ }^{1}$ Department of Colorectal Surgery, Ningbo Medical Center Lihuili Hospital, Ningbo, China \\ ${ }^{2}$ Department of Clinical Laboratory, Hwa Mei Hospital, University of Chinese Academy of Science (Ningbo No.2 Hospital), \\ Ningbo, China \\ ${ }^{3}$ Department of Radiology, Hwa Mei Hospital, University of Chinese Academy of Science (Ningbo No.2 Hospital), Ningbo, \\ China
}

\begin{abstract}
Circular RNA-microRNA (circRNA-miR) node has recently been found to modulate cancer process. Here, we investigated whether circCDYL and miR-150-5p exerted biological function in colon cancer cells. Colon cancer tissues were collected and subjected to qRT-PCR assay for circCDYL and miR-150-5p. SW480 and SW620 cells were forced to overexpress circCDYL and miR150-5p before subjected to viability, colony formation, apoptosis, migration, invasion and protein (associated with proliferation, apoptosis and signaling pathways) assays. To confirm the combined function, the cells were transfected to simultaneously overexpress circCDYL and miR-150-5p. We found circCDYL was generally decreased while miR-150-5p was increased in colon cancer tissues in parallel with the para-carcinoma tissues. In circCDYL-transfected SW480 and SW620 cells, circCDYL decreased viability and promoted apoptosis with down-regulation of c-Myc and cyclin D1, up-regulation of p53, and cleavage of caspase- 3 and PARP. Besides, migration and invasion behaviors were impeded. By contrast, miR-150-5p showed a carcinogenesis. However, suppressive role of circCDYL in cellular growth and migration was restrained in the cells simultaneously transfected with circCDYL and miR-150-5p, which was companied by down-regulation of PTEN and phosphorylation of PI3K, AKT, JAK2 and STAT5. circCDYL overexpression repressed cellular growth and migration via repressing miR-150-5p in colon cancer cells.
\end{abstract}

Key words: circCDYL - microRNA-150-5p - Colon cancer - PI3K/AKT/JAK/STAT

\section{Introduction}

Colorectal cancer is the second most frequent causative factor of cancer death globally but third in terms of incidences (Bray et al. 2018). It has been estimated that over 1.8 million new cases and 0.88 million deaths occur in 2018 (Bray et al. 2018). Although most colorectal cancers are considered sporadic, the etiology has been ascribed to inherited cancer syndromes, inflammation conditions and industrialized lifestyles (Sierra and Forman 2016). Colorectal cancer process is accompanied by genetic alterations, including not

Correspondence to: Hao Gu, Department of Radiology, Hwa Mei Hospital, University of Chinese Academy of Science (Ningbo No.2 Hospital), No.41 XiBei Street, Ningbo 315010, Zhejiang, China E-mail: guhao47gh@163.com only oncogenes and tumor suppressor genes (Carethers and Jung 2015), but also noncoding RNA, for instance, long non-coding RNA (lncRNA) (Xie et al. 2016), microRNA (miRNA) (Slattery et al. 2016) and circular RNA (circRNA) (Zhang et al. 2017). A subset of these noncoding RNA were considered as hallmarks of cancers and were potential targets for therapies because of their clinical signatures and biological functions (Iguchi et al. 2015; Pellatt et al. 2016; Zhang et al. 2017).

circRNAs are typical of covalently closed loop without a 5'-cap or 3'- Poly A tail which are more stable than linear RNAs. Some of them function as scaffolds in protein complex assembly (Du et al. 2017), mediate subcellular localization of proteins (Armakola et al. 2012), regulate parental gene expression ( $\mathrm{Li}$ et al. 2015b), and sponge miRNAs (Li et al. 2015a). Recently, it has been underlined that circRNA- 
miRNA-mRNA axis is involved in a multitude of disease pathways, suggesting this association modulates gene expression in malignant tumors (Rong et al. 2017, Verduci et al. 2019). Circular RNA CDYL (circCDYL) is produced by exon4 spliceosome-mediated pre-mRNA splicing and mainly localizes in cytoplasm (Sun et al. 2019). circCDYL has been detected overexpressed in hepatocellular carcinoma and bladder cancer tissues (Sun et al. 2019; Wei et al. 2019). A nearest study disclosed that circCDYL sponges miRNAs to modulate gene expression in hepatocellular carcinoma (Wei et al. 2019), which inspired us to examine the level of circCDYL in colon cancers and its biological role in colon cancer cells.

miRNA is composed of $10-25$ nucleotides and is sophistically processed. Mature miRNAs target mRNAs and meditate the expression of genes accounting for one-third of human genome in a posttranscriptional manner (Babashah and Soleimani 2011). In the last few years, emerging evidences have heighted their significance in the development and progression of malignant tumors (Reddy 2015). An antecedent study has reported that microRNA-150-5p (miR-150-5p) exacerbates cellular metastasis in non-small cell lung cancer ( $\mathrm{Li}$ et al. 2016). Its role in the progression of colorectal cancer has also been explored (Aherne et al. 2015; Sarlinova et al. 2016). Deserved to be mentioned, miR-150-5p is sponged by circular RNA ZNF609 (a competitive endogenous RNA) to mediate AKT3 expression in Hirschsprung's disease (Peng et al. 2017). As a consequence, there might be a possibility that circCDYL sponges miR$150-5 p$ in colon cancer cells.

Here, we firstly detected circCDYL in colon cancer tissues. Continually, we investigated the gain-of-function of circCDYL in colon cancer cell lines (SW480 and SW620).

Table 1. Clinical pathological parameters (gender, age, tumor size, grade, and stage) from 25 patients diagnosed with colorectal cancer

\begin{tabular}{llc}
\hline Variables & & Case number \\
\hline \multirow{2}{*}{ Gender } & male & 18 \\
& female & 7 \\
\cline { 2 - 3 } Age & $\leq 50$ & 6 \\
(years) & $50-70$ & 14 \\
& $>70$ & 5 \\
\cline { 2 - 3 } Tumor size & $\leq 3$ & 5 \\
$(\mathrm{~cm})$ & $3-5$ & 16 \\
& $>5$ & 4 \\
Histological & I grade (well) & 3 \\
grade & II grade (moderate) & 16 \\
& III grade (poor) & 3 \\
\cline { 2 - 3 } Tumor & I-II & 14 \\
stage & III-IV & 11 \\
\hline
\end{tabular}

At last, circCDYL-miR-150-5p axis was probed in cellular progression of colon cancer cells. In addition, signaling cascades involved in cancers were focused.

\section{Materials and Methods}

\section{Tissue collection and cell culture}

To evaluate circCDYL level in colon cancer tissues, clinical colon cancer tissues were collected from patients $(n=25)$ pathologically diagnosed with colorectal cancer and hospitalized at Ningbo Medical Center Lihuili Hospital (Ningbo, China). The pathological features including gender, age, tumor size, grade, and stage were presented in Table 1. The colon cancer tissues were excised by surgery therapy. The paired surrounding normal tissues were excised as comparison. None of the patients received any therapies before. All experiments were carried out in compliance with the standard from the Ethics Committee of Ningbo Medical Center Lihuili Hospital. Informed consents from human subjects were obtained for this study.

Human colon cancer cell lines SW480 (Catalog No. C0009001; Addexbio, San Diego, CA, USA) and SW620 (CCL-227; American Type Culture Collection, ATCC, Rockville, MD, USA) were maintained in Leibovitz's L-15 medium in addition with $10 \%$ fetal bovine serum (Gibco, Gaithersburg, $\mathrm{MD}, \mathrm{USA}$ ) at $37^{\circ} \mathrm{C}$ in an incubator containing $100 \%$ air.

\section{Transfection}

To up-regulate circCDYL, circCDYL sequence was cloned into pLCDH-circRNA vector by Ribobio (Guangzhou, China). miR-150-5p mimic and negative control mimic (NC mimic) were synthesized by Life Technologies (Waltham, MA, USA). Transfection was performed using Lipofectamine 3000 in compliance with the manufacturer's description (Invitrogen, Carlsbad, CA, USA). The cells were collected after transfection for $48 \mathrm{~h}$ for confirmation by qRT-PCR.

\section{Quantification of circCDYL and miR-150-5p}

Whole cellular RNA from colon cancer tissues and colon cancer cell lines (SW480 and SW620) was isolated by Trizol reagent kit (Invitrogen) in compliance with the user's protocol. Reverse transcription RNA was carried out with $2 \mu \mathrm{g}$ of total RNA, random primers and HiScript II Q Select RT Supermix Kit from Vazyme Biotech (Nanjing, China) to synthesize cDNA. Quantitative real-time PCR was conducted on ABI 7300 SYSTEM (Applied Biosystems, Foster City, CA, USA) with specific primers for circCDYL 
(Forward 5'-CTTAGCTGTTAACGGGAAA-3'; Reverse 5'-CTGTTGAAGTCGTGGATGT-3') and miR-150-5p (Forward 5'-ACACTCCAGCTGGGTCTCCCAACCCTTGTA-3'; Reverse 5'-CTCAACTGGTGTCGTGGAGTCGGCAATTCAGTTGAGCACTGGTA-3') using Power SYBR Green Master Mix (Applied Biosystems). Relative expression of circCDYL and miR-150-5p was normalized to GAPDH (Forward 5'-GCACCGTCAAGGCTGAGAAC-3'; Reverse 5'-GGATCTCGCTCCTGGAAGATG-3') and U6 (Forward 5'-CTCGCTTCGGCAGCACA-3'; Reverse 5'-AACGCTTCACGAATTTGCGT-3'), respectively, and was calculated with $2^{-\Delta \Delta C t}$ method.

\section{Cell viability}

The cells were inoculated in a 96-well plate with a density of $1 \times 10^{3}$ cells each well. After maintained for $24 \mathrm{~h}$, the culture was added with CCK-8 reagent (adding $10 \mu \mathrm{l}$ ) (Dojindo Molecular Technologies, Kumamoto, Japan) and incubated for $1 \mathrm{~h}$ at $37^{\circ} \mathrm{C}$. At last, the absorbance was detected using Microplate Reader at $450 \mathrm{~nm}$ (Molecular devices, San Jose, CA, USA).

\section{Colony formation assay}

The cells were grown onto a 6-well plate with a density of $2 \times 10^{2}$ cells per well and maintained at $37^{\circ} \mathrm{C}$ for two weeks. After washed in phosphate buffered saline (Sigma-Aldrich, St. Louis, MO, USA), the cells were stained by crystal violet (Sigma-Aldrich). The colonies consisting of over 50 cells were accepted under Olympus IX81 microscope (Olympus, Tokyo, Japan).

\section{Apoptosis assay}

Apoptosis in cultured cells were observed using flow cytometric analysis. In short, the cells were doubly stained in Annexin V-FITC and propidium iodide (PI) kit (BD Biosciences, Franklin Lakes, NJ, USA) in compliance with manufacturer's instruction. Finally, the cells were observed using BD FASCalibur apparatus (BD Biosciences). The cells at early (Annexin V-FITC+/PI-) and late (Annexin VFITC+/PI+) stages of apoptosis were included and counted but necrotic cells (Annexin V-FITC-/PI+) or viable cells (Annexin-FITC-/PI-).

\section{Migration and invasion assay}

Transwell insets (BD Biosciences) were used to evaluate the invasion and migration activities of cells. For migration assay, the cell suspension $\left(2 \times 10^{5}\right.$ cells in serum-free medium) was grown in to upper chamber and maintained for $48 \mathrm{~h}$. The non-migratory cells were removed, and the cells under the membrane were fixed with methanol before stained by crystal violet. As for invasion activity, the chamber was pre-coated using matrigel matrix (BD Biosciences). Lastly, the cells in the lower chamber were counted using a microscope.

\section{Western blot}

Cells were harvested and subjected to protein extraction in cell lysis buffer (Beyotime, Shanghai, China). After centrifugation $\left(14,000 \times \mathrm{g}, 5 \mathrm{~min}, 4^{\circ} \mathrm{C}\right)$, the obtained proteins $(15-30 \mu \mathrm{g})$ were loaded onto sodium dodecyl sulfate polyacrylamide gel electrophoresis to separate proteins which were then transferred onto a nitrocellulose membrane (Millipore, Bedford, MA, USA). After blocked in 5\% non-fat milk, the proteins were hybridized with primary antibodies against AKT (1:1,000; GTX128415) (GeneTex, Irvine, CA, USA), pSer473 AKT ( $1 \mu \mathrm{g} / \mathrm{ml}$; GTX59559), caspase-3 (1 $\mu \mathrm{g} / \mathrm{ml} ; 3138)$ (BioVision, Milpitas, CA, USA), PARP ( $1 \mu \mathrm{g} / \mathrm{ml} ; 3002)$, PI3K $(1 \mu \mathrm{g} / \mathrm{ml} ; 3959)$, c-Myc (1:1,000; F47610) (NSJ Bioreagents, San Diego, CA, USA), p53 (1 $\mu \mathrm{g} / \mathrm{ml}$; R31441), Cyclin D (1:1,000; orb99429) (Biorbyt, Cambridge, UK), JAK2 (1:1,000; orb14527), PTEN (1:1,000; orb228972), pTyr467 PI3K (1:1,000; orb14998), STAT5 (1:1,000; orb224180), pY694/699 STAT5 (1:1,000; orb224181), and pTyr1007 JAK2 (1:1,000; AHP2639; Bio-Rad, Hercules, CA, USA) with $\beta$-actin as a loading control (1:1,000; ab8227; Abcam, Cambridge, UK) at $4^{\circ} \mathrm{C}$ overnight. The primary antibodies were probed by goat anti-rat IgG conjugated by horseradish peroxidase (HRP) (1:5,000; ab97057; Abcam) for $1 \mathrm{~h}$ at room temperature. Then, the membranes were transferred onto Bio-Rad ChemiDoc XRS system (Bio-Rad) and supplemented with chemiluminescent HRP substrate (Millipore). Western blots corresponded to a representative performance of triple experiments.

\section{Data analysis}

Data analysis was conducted with GraphPad Prism 6.0 software (GraphPad, San Diego, CA, USA). Student's $t$-test was used to compare the difference between two groups. One way analysis of variance followed by Bonferroni's post-test was performed to compare the difference among all groups. Data were expressed as the mean \pm standard deviation. The $p$-values less than 0.05 were considered to indicate a statistical significance. The differences were accepted when $p$ was less than 0.05 .

\section{Ethics approval and consent to participate.}

All procedures performed in studies involving human participants were in accordance with the ethical standards 
of the institutional committee and with the 1964 Helsinki declaration and its later amendments or comparable ethical standards.

All experiments were carried out in compliance with the standard from the Ethics Committee of Ningbo Medical Center Lihuili Hospital. Written informed consents were obtained from patients before surgery.

\section{Results}

circCDYL was down-regulated in colon cancer and its overexpression repressed the growth and migration and invasion of human colon cell lines

To investigate the association between circCDYL and colon cancer, we examined circCDYL level in human colon tissues from 25 patients. We found circCDYL was decreased in tumor tissues relative to the corresponding adjacent tissues $(p<0.01)$ (Fig. 1A). Next, circCDYLoverexpressed SW480 and SW620 cells were constructed (both $p<0.001$ ) (Fig. 1B). We found the viability of SW480 and SW620 cells was reduced after circCDYL overexpression (both $p<0.01$ ) (Fig. 1C) compared with the un-transfected cells. Consistently, the formation of colony was significantly repressed (both $p<0.01$ ) in circCDYL-transfected cells compared with the un-transfected cells (Fig. 1D). Additionally, c-Myc and cyclin D1 were down-regulated in circCDYL-overexpressed cells (both $p<0.05)$ while $p 53$ was enhanced $(p<0.001)$ relative to the control cells (Fig. 1E). Apoptosis was observed to occur in circCDYL-transfected cells (both $p<0.001$ ) (Fig. $1 F)$, which was accompanied by the enhanced cleaved process of caspase- 3 and PARP (both $p<0.001$ ) (Fig. $1 G)$. Collectively, circCDYL overexpression suppressed the growth of colon cancer cells.

To evaluate the role of circCDYL in migration and invasion, we dissected the alteration of migration and invasion behaviors. After transfection, circCDYL-overexpressed SW480 and SW620 cells were subjected to the assay using transwell inserts. As shown in Figure $1 \mathrm{H}$ and $\mathrm{I}$, we found the migration and invasion (both $p<0.01$ ) activities were significantly weakened in circCDYL-transfected SW480 and SW620 cells. Taken together, migration and invasion activities were retarded by circCDYL in colon cancer cells.

\section{miR-150-5p was enriched in colon cancer tissues and its upregulation promoted the growth and migration and invasion of colon cancer cells}

Besides, we detected the abundance of miR-150-5p in colon cancer tissues $(p<0.01)$ (Fig. 2A). To study the biological role of miR-150-5p, we constructed transfectants which overexpressed miR-150-5p. As shown in Figure 2B, miR$150-5 p$ was enhanced in SW480 $(p<0.001)$ and SW620 $(p<0.001)$ after transfected with miR-150-5p mimic. We found miR-150-5p elevated the viability and colony formation of SW480 and SW620 cells (both $p<0.05$ ) (Fig. 2C, D). Besides, miR-150-5p overexpressed cells indicated increases in $\mathrm{c}-\mathrm{Myc}(p<0.05$ or $p<0.01)$ and Cyclin D1 $(p<0.05$ or $p<0.01)$ while a decrease in p53 (both $p<$ 0.05) (Fig. 2E). Meanwhile, miR-150-5p overexpression conferred an inhibitory role in apoptosis (both $p<0.05$ ) as depicted in Figure 2F. Results from Western blot showed miR-150-5p blocked the cleavage of caspase- 3 and PARP (both $p<0.01$ ), further corroborated this finding (Fig. $2 \mathrm{G})$. In addition, the migratory and invasive activities were elevated in SW480 and SW620 cells transfected with miR-150-5p mimic (both $p<0.05$ ) (Fig. 2H, I). Summarily, miR-150-5p induced the growth and migration and invasion of colon cancer cells.

circCDYL down-regulated miR-150-5p to buffer the growth and migration and invasion of colon cancer cells

Meanwhile, miR-150-5p was down-regulated in circCDYLoverexpressed SW480 $(p<0.01)$ and SW620 $(p<0.01)$ cells (Fig. 3A). This mediatory connection promoted us to wonder whether miR-150-5p functions as a mediator of circCDYL. Herein, we conjectured that miR-150-5p up-regulation might preclude the suppressive activity of circCDYL. As expected, the viability (Fig. 3B) and colony formation (Fig. 3C) were enhanced at least to a degree (both $p<0.05$ ) in circCDYL- and miR-150-5p- overexpressed SW480 and SW620 cells compared with circCDYL-overexpressed cells. Additionally, miR-150-5p resulted in the abundance of cMyc (both $p<0.05$ ) and cyclin D1 (both $p<0.05$ ) and repressed the accumulation of $\mathrm{p} 53$ (both $p<0.01$ ) (Fig. 3D) in the cells transfected with circCDYL and miR-150-5p relative to the cells only transfected with circCDYL. What's more, miR-150-5p enhanced the ability of SW 480 and SW620 cells to resist circCDYL overexpression-induced apoptosis (both $p<0.05)$ (Fig. 3E). Uniformly, circCDYL-triggered cleavage of caspase- 3 and PARP was impeded by miR-150-5p (both $p<0.01$ ) (Fig. 3F). Summarily, circCDYL antagonized the growth of colon cancer cells in conjunction with miR-150$5 \mathrm{p}$ down-regulation.

To verify that miR-150-5p was implicated in the modulation of migration and invasion in circCDYL-overexpressed cells, we simultaneously up-regulated circCDYL and miR150-5p by transfection. Subsequently, the cells were subjected to migration and invasion assays. As shown in Figure 3G and $\mathrm{H}$, miR-150-5p obviously accelerated the migration and invasion behaviors (both $p<0.05$ ). As a whole, circCDYL overexpression might impede migration and invasion via down-regulating miR-150-5p. 
miR-150-5p activated PI3K/AKT and JAK/STAT cascades blunted by circCDYL

To explore the related mechanisms, we further dissected the alteration of PI3K/AKT and JAK/STAT pathways. As depicted in Figure 4A, circCDYL overexpression promoted the production of PTEN $(p<0.01$ or $p<0.001)$, while repressed the phosphorylation of PI3K (both $p<$ 0.01 ) and AKT (both $p<0.01$ ). However, we observed this modulatory effect of circCDYL was negated in SW480 and SW620 cells simultaneously transfected with circCDYL and miR-150-5p $(p<0.05$ or $p<0.01)$ compared with the cells only transfected with circCDYL. As for JAK/STAT, the phosphorylation of JAK2 and STAT5 $(p<0.01$ or $p<$ $0.001)$ was notably impeded in circCDYL-overexpressed cells while this phosphorylated expression of JAK2 and STAT5 was significantly induced $(p<0.05$ or $p<0.01)$ in SW480 and SW620 cells simultaneously overexpressing circCDYL and miR-150-5p (Fig. 4B). Consequently, miR-150-5p down-regulation might be indispensable for circCDYL-elicited blockage of PI3K/AKT and JAK/STAT transduction cascades.

\section{Discussion}

Recent studies demonstrated circRNA was involved in the pathogenesis of colon cancer via modulating oncogenic expression (Hsiao et al. 2017). A subset of researches was performed to address the underlying mechanisms which might be associated with its role as a competing endogenous RNA (Zhong et al. 2018). Recently, a study in colon cancer, showed that a circRNA exacerbates the growth and metastasis by sponging miRNA, which highlights their significance in malignant tumors (Xu et al. 2017). In this study, we focused on the the gain-of-function of circCDYL in colon cancer cells as well as the function of miR-150-5p during this process.

circCDYL has been detected at a high level in the nonmalignant bladder cancer cells relative to the metastatic bladder cancer cells (Okholm et al. 2017; Sun et al. 2019). However, it is still largely unexplored about the causative factor of its ectopic expression. In line with the abovementioned results, we also detected circCDYL in 25 pairs of colon cancer tissues and para-carcinoma tissues, and the down-regulation of circCDYL was observed in colon cancer tissues. Kaplan-Meier survival analysis has been carried out and implied patients with a high circCDYL level are at a lower risk of bladder cancer advance relative to patients with a low expression of circCDYL (Okholm et al. 2017). These results suggested that circCDYL is a prognostic biomarker potential and a promising target candidate for malignant tumors, such as colon and bladder cancers.
The investigation about its biological role in colon cancer proved that circCDYL overexpression caused viability defects, reduced colony formation, impeded migration and invasion behaviors and facilitated apoptosis. Similarly, circCDYL overexpression suppressed cellular growth and migration in bladder cancer (Sun et al. 2019). Besides, we found the protein level of c-Myc and cyclin D1 was reduced in circCDYL-transfected SW480 and SW620 cells, while p53 was elevated. Studies found c-Myc promotes cell-cycle from G0-G1 phase to $S$ phase, while circCDYL overexpression negates this process via repressing c-Myc expression (Sun et al. 2019). In addition, circCDYL induced the cleavage of caspase-3 which is accountable for genome instability in tumor formation (Liu et al. 2015). circCDYL also impeded the activation of PARP which is overexpressed in a multitude of cancers and implicated in DNA damage (Kummar et al. 2012). Based on this biological function, circCDYL was considered as a promising candidate for colon cancers.

miR-150 has been reported to be up-regulated in multitude of cancers (Wu et al. 2010; Zhang et al. 2013). miR150 was also reported in colon cancers and implicated in the development of rectal cancers (Ma et al. 2012; Aherne et al. 2015). Intriguingly, studies found miR-150-5p is modulated by circRNA in hirschsprung disease (Zhou and Yu 2017), myocardial fibrosis (Zhou et al. 2017) as well as cervical cancer (Cai et al. 2019). Here miR-150-5p was observed to be abundant in colon cancer tissues. Besides, miR-150-5p overexpression provided growth advantages for colon cancer cells. Consistently, we found both circCDYL down-regulation and miR-150-5p up-regulation showed a carcinogenesis in colon cancer cells SW480 and SW620 (SW480 and SW620).

Of note, we found miR-150-5p overexpression abolished the suppressive properties of circCDYL in the growth and migration behaviors of colon cancer cells, suggesting miR150-5p might emerge as a downstream target of circCDYL. A previous study has found that miR-150-5p participates in cancer process by targeting the expression of genes, like membrane-type-1 matrix metalloproteinase (Sakr et al. 2016). In our study, we found miR-15-5p overexpression restored c-Myc and Cyclin D1 expression, while repressed p53 which has be reported targeted by miR-150 to facilitate proliferation of lung cancer cells (Zhang et al. 2013). As a consequence, we considered that miR-150-5p functioned as a mediator of circCDYL to be down-regulated for mediating gene expression implicated cancer process.

Furthermore, we found circCDYL overexpression upregulated PTEN and triggered phosphorylation of PI3K and AKT, while miR-150-5p overexpression inactivated PI3K/AKT pathway. Besides, the activation of JAK/STAT was blocked by circCDYL. By contrast, miR-150-5p overexpression triggered the activation of JAK/STAT pathway. 


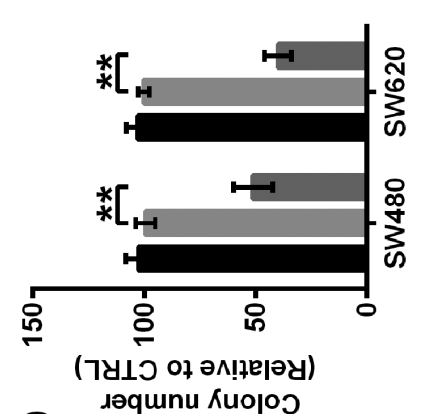

口

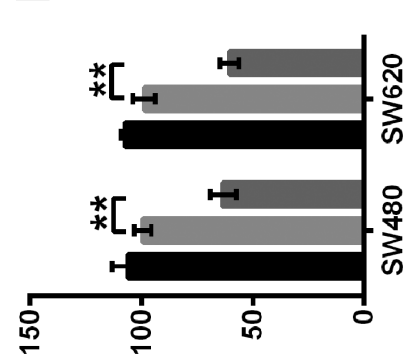

0

(\%) К‼!q!! ||әว

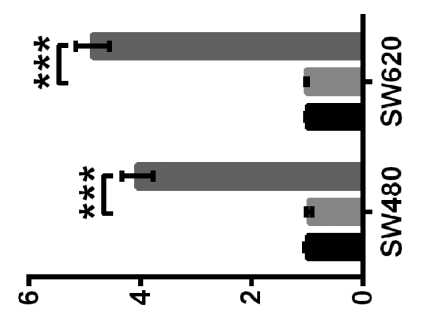

m

(9ก 아 әм!ฺе әәу)
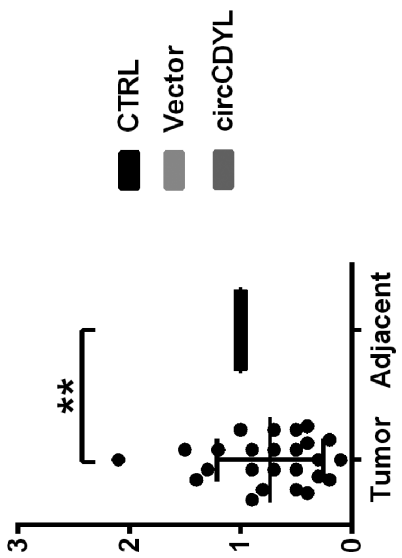

(9n 아 әм!ре|әу)

$<$

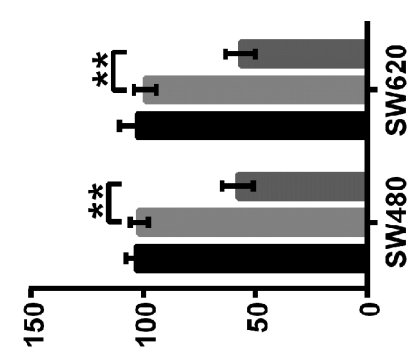

工 (\%) иопџелб!и әм!ฺерәу
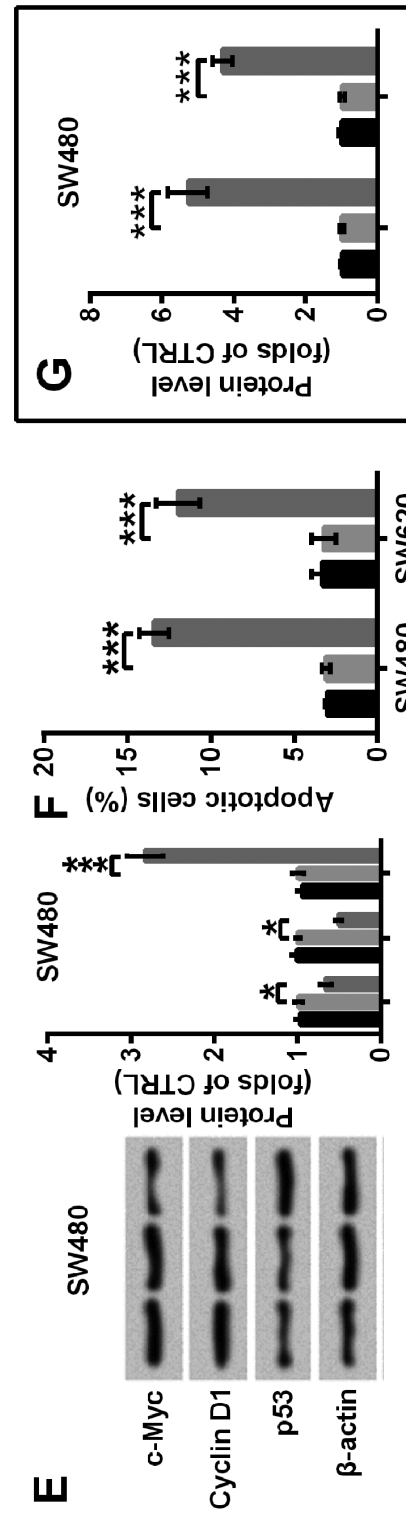

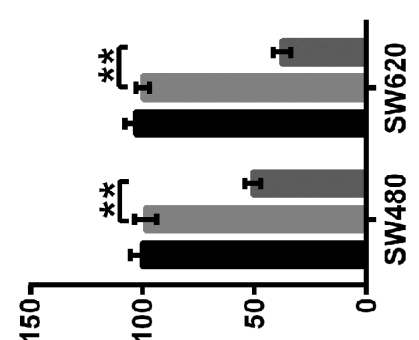

(\%) ио!senu! әм!̣е|әу
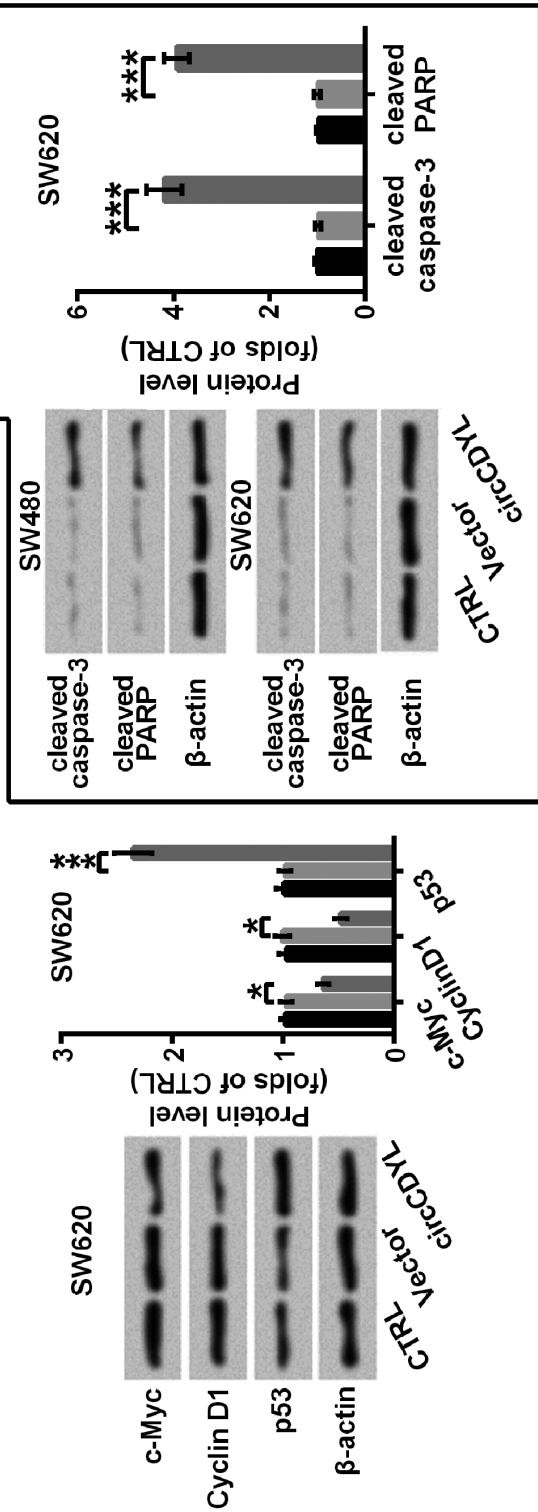

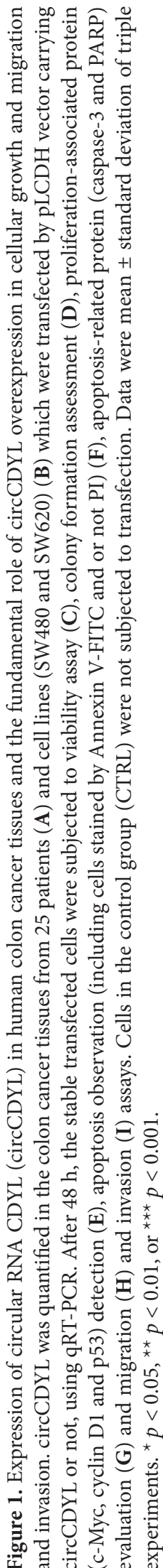



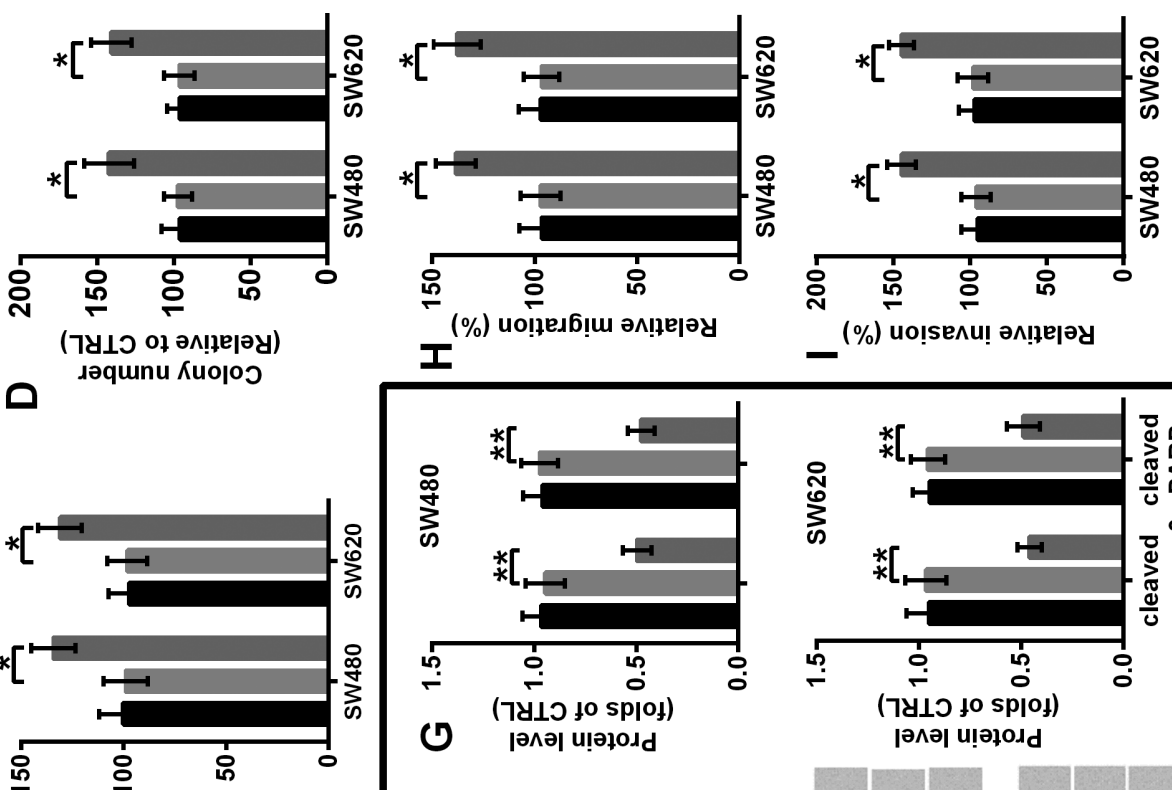

ง (\%) К‼!q!!^ \|әว

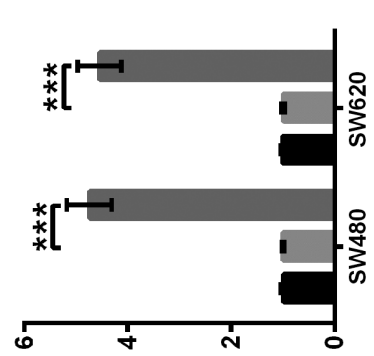

$I$
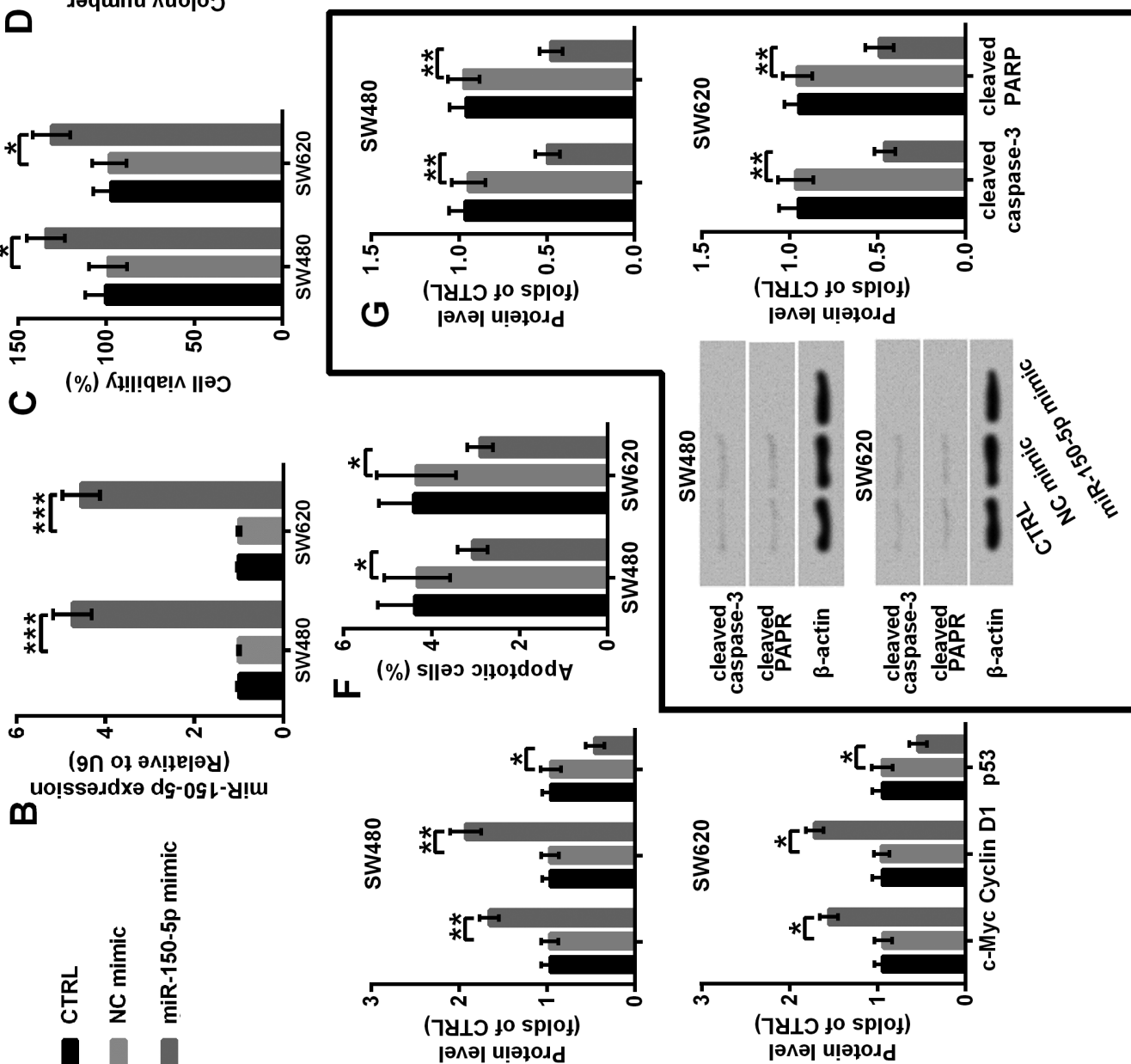

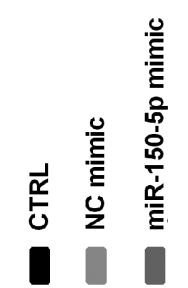
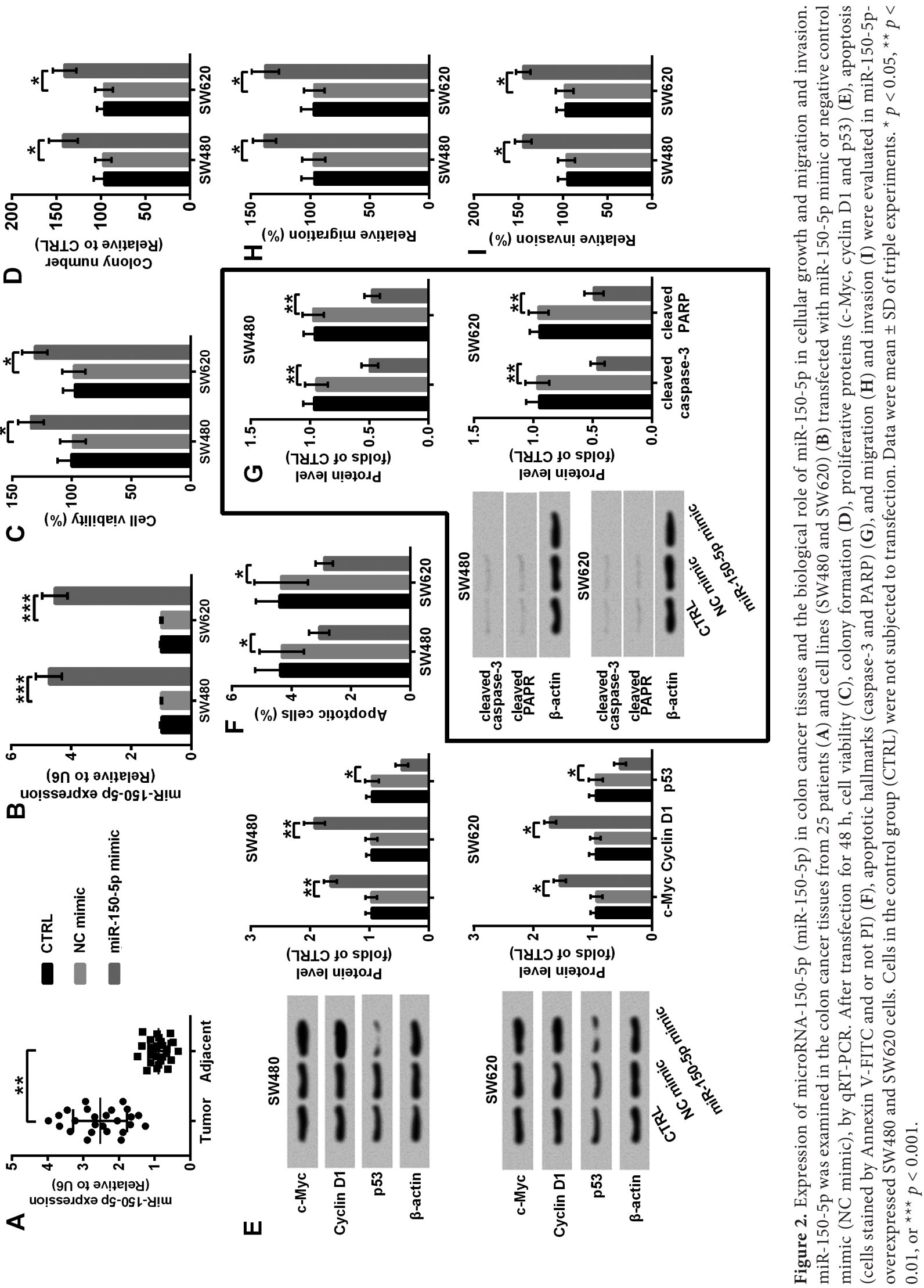

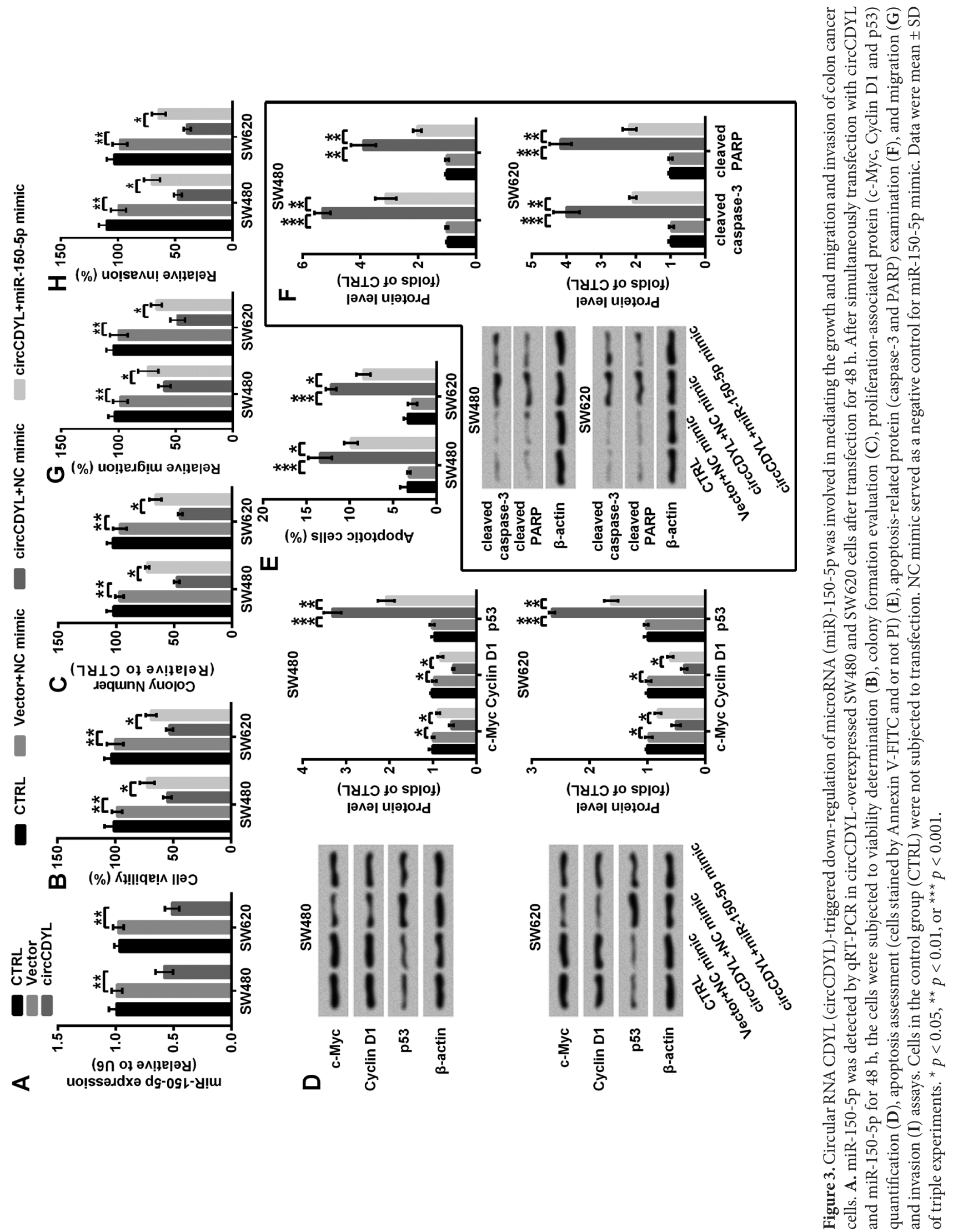
PI3K/AKT and JAK/STAT pathways have been confirmed as potential targets for colorectal cancer (Pandurangan 2013, Slattery et al. 2013). As a result, circCDYL overexpression indirectly caused the bluntness of PI3K/AKT and JAK/ STAT via repressing the expression of miR-150-5p, and consequently exerted an antitumor role in colon cancer cells.

Collectively, circCDYL was generally up-regulated while miR-150-5p was down-regulated in colon cancer tissues in parallel with the para-cancerous tissues. The growth and migration of colon cancer cells were significantly impeded after transfection with circCDYL. By contrast, the cells showed a growth advantage after miR-150-5p overexpression was induced. Of note, the growth and migration were promoted with blockage of PI3K/AKT and JAK/ STAT pathways in the cells simultaneous overexpressing circCDYL and miR-150-5p compared with the cells only overexpressing circCDYL.

Acknowledgements. This work was supported by the Key Subjects of Ningbo No.2 Hospital (2016004/2016009).
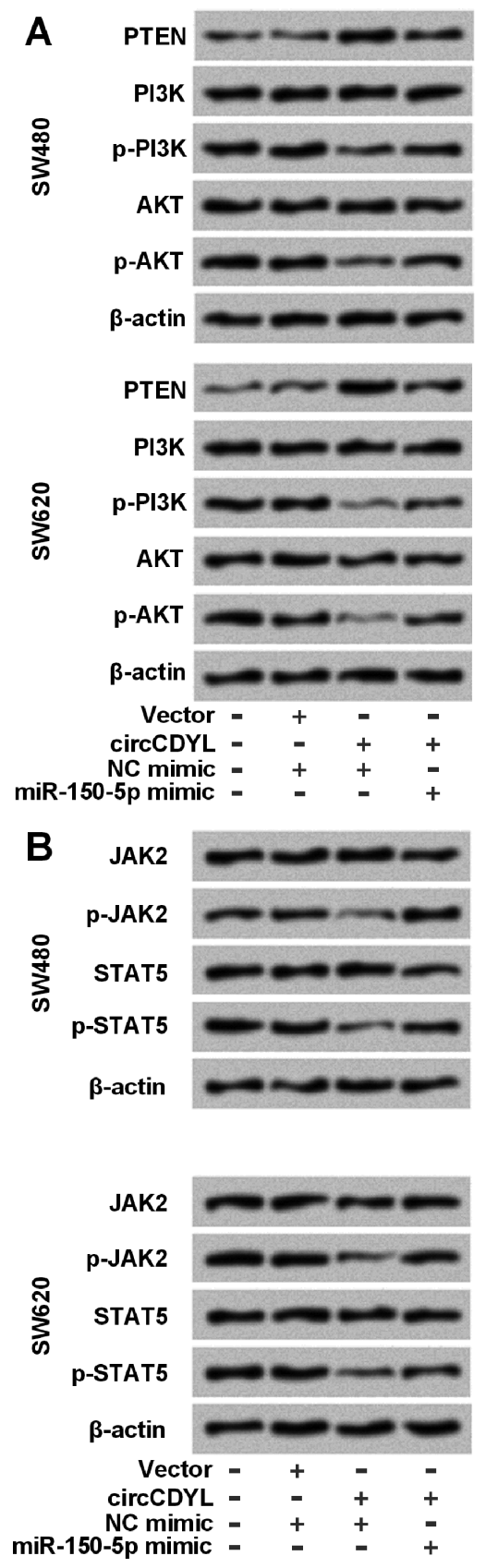
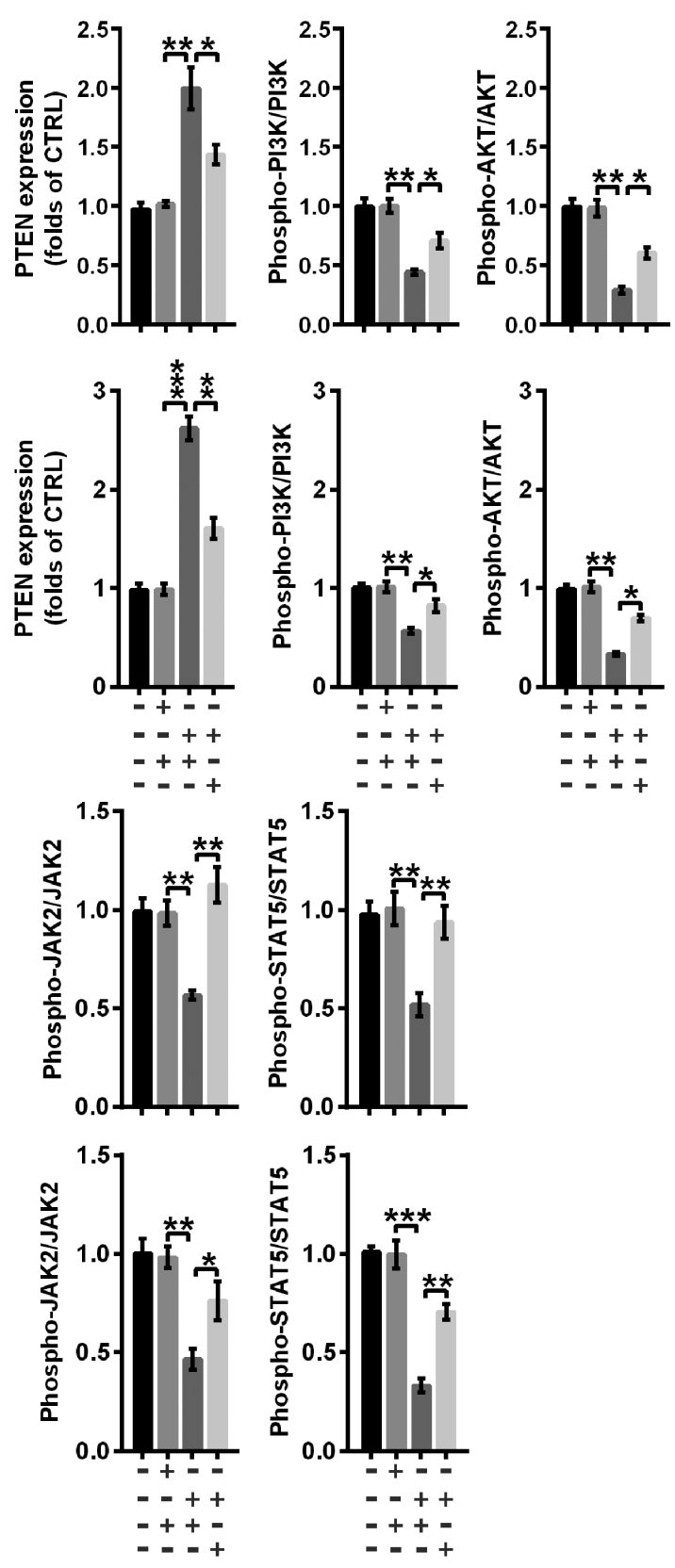

Figure 4. Circular RNA CDYL (circCDYL) blocked activation of PI3K/AKT and JAK/STAT cascades in dependent on the down-regulation of microRNA (miR)-150-5p in colon cancer cells. After simultaneous transfection with circCDYL and miR150-5p for $48 \mathrm{~h}$, Western blot assay was performed to evaluate the activation of PI3K/AKT (A) and JAK/STAT (B) pathways using the indicated antibodies against the associated regulators. Cells in the control group (CTRL) were not subjected to transfection. NC mimic served as a negative control for miR150-5p mimic. Data were mean \pm SD of triple experiments. ${ }^{*} p<$ $0.05,{ }^{* *} p<0.01$, or ${ }^{* * *} p<0.001$. 
Conflict of interest. The authors declare that they have no competing interests. This research did not receive any specific grant from funding agencies in the public, commercial, or not-for-profit sectors.

Authors' contributions. Conceived and designed the experiments: Hao Gu; performed the experiments and analyzed the data: Wei Cui, Jinhua Dai, Jianbo Ma; wrote the manuscript: Wei Cui, Hao Gu.

\section{References}

Aherne ST, Madden SF, Hughes DJ, Pardini B, Naccarati A, Levy M, Vodicka P, Neary P, Dowling P, Clynes M (2015): Circulating miRNAs miR-34a and miR-150 associated with colorectal cancer progression. BMC Cancer 15, 329

https://doi.org/10.1186/s12885-015-1327-5

Armakola M, Higgins MJ, Figley MD, Barmada SJ, Scarborough EA, Diaz Z, Fang X, Shorter J, Krogan NJ, Finkbeiner S, et al. (2012): Inhibition of RNA lariat debranching enzyme suppresses TDP-43 toxicity in ALS disease models. Nat. Genet. 44, 1302-1309 https://doi.org/10.1038/ng.2434

Babashah S, Soleimani M (2011): The oncogenic and tumour suppressive roles of microRNAs in cancer and apoptosis. Eur. J. Cancer (Oxford, England) 47, 1127-1137 https://doi.org/10.1016/j.ejca.2011.02.008

Bray F, Ferlay J, Soerjomataram I, Siegel RL, Torre LA, Jemal A (2018): Global cancer statistics 2018: GLOBOCAN estimates of incidence and mortality worldwide for 36 cancers in 185 countries. CA Cancer J. Clin. 68, 394-424 https://doi.org/10.3322/caac.21492

Cai H, Zhang P, Xu M, Yan L, Liu N, Wu X (2019): Circular RNA hsa_circ_0000263 participates in cervical cancer development by regulating target gene of miR-150-5p. J. Cell. Physiol. 234, 11391-11400 https://doi.org/10.1002/jcp.27796

Carethers JM, Jung BH (2015): Genetics and genetic biomarkers in sporadic colorectal cancer. Gastroenterol. 149, 1177-1190 https://doi.org/10.1053/j.gastro.2015.06.047

Du WW, Fang L, Yang W, Wu N, Awan FM, Yang Z, Yang BB (2017): Induction of tumor apoptosis through a circular RNA enhancing Foxo3 activity. Cell Death Differ. 24, 357-370 https://doi.org/10.1038/cdd.2016.133

Hsiao KY, Lin YC, Gupta SK, Chang N, Yen L, Sun HS, Tsai SJ (2017): Noncoding effects of circular RNA CCDC66 promote colon cancer growth and metastasis. Cancer Res. 77, 2339-2350 https://doi.org/10.1158/0008-5472.CAN-16-1883

Iguchi T, Uchi R, Nambara S, Saito T, Komatsu H, Hirata H, Ueda M, Sakimura S, Takano Y, Kurashige J, et al. (2015): A long noncoding RNA, lncRNA-ATB, is involved in the progression and prognosis of colorectal cancer. Anticancer Res. 35, 1385-1388

Kummar S, Chen A, Parchment RE, Kinders RJ, Ji J, Tomaszewski JE, Doroshow JH (2012): Advances in using PARP inhibitors to treat cancer. BMC Med. 10, 25 https://doi.org/10.1186/1741-7015-10-25
Li F, Zhang L, Li W, Deng J, Zheng J, An M, Lu J, Zhou Y (2015a): Circular RNA ITCH has inhibitory effect on ESCC by suppressing the Wnt/beta-catenin pathway. Oncotarget 6, 60016013 https://doi.org/10.18632/oncotarget.3469

Li H, Ouyang R, Wang Z, Zhou W, Chen H, Jiang Y, Zhang Y, Li H, Liao M, Wang W, et al. (2016): MiR-150 promotes cellular metastasis in non-small cell lung cancer by targeting FOXO4. Sci. Rep. 6, 39001 https://doi.org/10.1038/srep39001

Li Z, Huang C, Bao C, Chen L, Lin M, Wang X, Zhong G, Yu B, Hu W, Dai L, et al. (2015b): Exon-intron circular RNAs regulate transcription in the nucleus. Nat. Struct. Mol. Biol. 22, 256-264 https://doi.org/10.1038/nsmb.2959

Liu X, He Y, Li F, Huang Q, Kato TA, Hall RP, Li CY (2015): Caspase-3 promotes genetic instability and carcinogenesis. Mol. Cell 58, 284-296 https://doi.org/10.1016/j.molcel.2015.03.003

Ma Y, Zhang P, Wang F, Zhang H, Yang J, Peng J, Liu W, Qin H (2012): miR-150 as a potential biomarker associated with prognosis and therapeutic outcome in colorectal cancer. Gut 61, 1447-1453 https://doi.org/10.1136/gutjnl-2011-301122

Okholm TLH, Nielsen MM, Hamilton MP, Christensen LL, Vang S, Hedegaard J, Hansen TB, Kjems J, Dyrskjot L, Pedersen JS (2017): Circular RNA expression is abundant and correlated to aggressiveness in early-stage bladder cancer. NPJ Genom. Med. 2, 36 https://doi.org/10.1038/s41525-017-0038-z

Pandurangan AK (2013): Potential targets for prevention of colorectal cancer: a focus on PI3K/Akt/mTOR and Wnt pathways. Asian Pac. J. Cancer Prev. 14, 2201-2205 https://doi.org/10.7314/APJCP.2013.14.4.2201

Pellatt DF, Stevens JR, Wolff RK, Mullany LE, Herrick JS, Samowitz W, Slattery ML (2016): Expression profiles of mirna subsets distinguish human colorectal carcinoma and normal colonic mucosa. Clin. Transl. Gastroenterol. 7, e152 https://doi.org/10.1038/ctg.2016.11

Peng L, Chen G, Zhu Z, Shen Z, Du C, Zang R, Su Y, Xie H, Li H, Xu X, Xia Y, Tang W (2017): Circular RNA ZNF609 functions as a competitive endogenous RNA to regulate AKT3 expression by sponging miR-150-5p in Hirschsprung's disease. Oncotarget 8, 808-818 https://doi.org/10.18632/oncotarget.13656

Reddy KB (2015): MicroRNA (miRNA) in cancer. Cancer Cell Int. 15, 38 https://doi.org/10.1186/s12935-015-0185-1

Rong D, Sun H, Li Z, Liu S, Dong C, Fu K, Tang W, Cao H (2017): An emerging function of circRNA-miRNAs-mRNA axis in human diseases. Oncotarget 8, 73271-73281 https://doi.org/10.18632/oncotarget.19154

Sakr M, Takino T, Sabit H, Nakada M, Li Z, Sato H (2016): miR150-5p and miR-133a suppress glioma cell proliferation and migration through targeting membrane-type-1 matrix metalloproteinase. Gene 587, 155-162 https://doi.org/10.1016/j.gene.2016.04.058

Sarlinova M, Halasa M, Mistuna D, Musak L, Iliev R, Slaby O, Mazuchova J, Valentova V, Plank L, Halasova E (2016): miR-21, 
miR-221 and miR-150 are deregulated in peripheral blood of patients with colorectal cancer. Anticancer Res. 36, 5449-5454 https://doi.org/10.21873/anticanres.11124

Sierra MS, Forman D (2016): Etiology of colorectal cancer (C1820) in Central and South America. Cancer in Central and South America. Lyon: International Agency for Research on Cancer https://doi.org/10.1016/j.canep.2016.03.010

Slattery ML, Lundgreen A, Kadlubar SA, Bondurant KL, Wolff RK (2013): JAK/STAT/SOCS-signaling pathway and colon and rectal cancer. Mol. Carcinog. 52, 155-166 https://doi.org/10.1002/mc.21841

Slattery ML, Herrick JS, Pellatt DF, Stevens JR, Mullany LE, Wolff E, Hoffman MD, Samowitz WS, Wolff RK (2016): MicroRNA profiles in colorectal carcinomas, adenomas and normal colonic mucosa: variations in miRNA expression and disease progression. Carcinogenesis 37, 245-261 https://doi.org/10.1093/carcin/bgv249

Sun J, Zhang H, Tao D, Xie F, Liu F, Gu C, Wang M, Wang L, Jiang G, Wang Z, Xiao X (2019): CircCDYL inhibits the expression of C-MYC to suppress cell growth and migration in bladder cancer. Artif. Cells Nanomed. Biotechnol. 47, 1349-1356 https://doi.org/10.1080/21691401.2019.1596941

Verduci L, Strano S, Yarden Y, Blandino G (2019): The circRNAmicroRNA code: emerging implications for cancer diagnosis and treatment. Mol. Oncol. 13, 669-680

Wei Y, Chen X, Liang C, Ling Y, Yang X, Ye X, Zhang H, Yang P, Cui X, Ren Y, et al. (2019): A noncoding regulatory rnas network driven by circ-CDYL acts specifically in the early stages hepatocellular carcinoma. Hepatology (Baltimore, Md.) (in press) https://doi.org/10.1002/hep.30795

Wu Q, Jin H, Yang Z, Luo G, Lu Y, Li K, Ren G, Su T, Pan Y, Feng B, Xue Z, Wang X, Fan D (2010): MiR-150 promotes gastric cancer proliferation by negatively regulating the pro-apoptotic gene EGR2. Biochem. Biophys. Res. Commun. 392, 340-345 https://doi.org/10.1016/j.bbrc.2009.12.182

Xie X, Tang B, Xiao YF, Xie R, Li BS, Dong H, Zhou JY, Yang SM (2016): Long non-coding RNAs in colorectal cancer. Oncotarget 7, 5226-5239 https://doi.org/10.18632/oncotarget.6446

Xu XW, Zheng BA, Hu ZM, Qian ZY, Huang CJ, Liu XQ, Wu WD (2017): Circular RNA hsa_circ_000984 promotes colon cancer growth and metastasis by sponging miR-106b. Oncotarget $\mathbf{8}$, 91674-91683 https://doi.org/10.18632/oncotarget.21748

Zhang N, Wei X, Xu L (2013): miR-150 promotes the proliferation of lung cancer cells by targeting P53. FEBS Lett. 587, 2346-2351 https://doi.org/10.1016/j.febslet.2013.05.059

Zhang P, Zuo Z, Shang W, Wu A, Bi R, Wu J, Li S, Sun X, Jiang L (2017): Identification of differentially expressed circular RNAs in human colorectal cancer. Tumour Biol. 39, 1010428317694546 https://doi.org/10.1177/1010428317694546

Zhong Y, Du Y, Yang X, Mo Y, Fan C, Xiong F, Ren D, Ye X, Li C, Wang Y, et al. (2018): Circular RNAs function as ceRNAs to regulate and control human cancer progression. Mol. Cancer 17,79 https://doi.org/10.1186/s12943-018-0827-8

Zhou B, Yu JW (2017): A novel identified circular RNA, circRNA_010567, promotes myocardial fibrosis via suppressing miR-141 by targeting TGF-beta1. Biochem. Biophys. Res. Commun. 487, 769-775 https://doi.org/10.1016/j.bbrc.2017.04.044

Received: July 5, 2019

Final version accepted: September 5, 2019 